\title{
Weiterer Beitrag zur Kenntnis von Polypeptiden, an deren Aufbau d-Aminobuttersäure beteiligt ist. \\ Von
}

Emil Abderhalden und Hsing Lang Chang.

(Aus dem physiologischen Institute der Universität Halle a. S.)

(Der Redaktion zugegangen am 11. März 1912.)

Die Synthese von Polypeptiden, an deren Aufbau $\alpha$-Aminobuttersäure beteiligt ist, wurde aus verschiedenen Gründen in Angriff genommen. Einmal interessierte es uns, zu erfahren, wie sich die peptolytischen Fermente gegenüber derartigen Polypeptiden verhalten und vor allem, ob sich ein Unterschied zeigt, je nachdem d-oder l-Aminobuttersäure als Baustein von Polypeptiden auftritt. Es zeigte sich,1) daß Glycyl-d-aminobuttersäure von Hefepreßsaft gespalten wird, dagegen blieb Glycyll-aminobuttersäure unangegriffen. Glycyl-dl-aminobuttersäure wurde asymmetrisch gespalten. Glycyl-l-aminobuttersäure, dAminobuttersäure und Glykokoll blieben übrig. Aus dieser Beobachtung geht hervor, daß die d-Aminobuttersäure offenbar eine Konfiguration besitzt, auf die bestimmte Fermente eingestellt sind, während l-Aminobuttersäure die *ferment-fremde * Komponente darstellt.

Wir hofften ferner durch eingehende Studien der $\alpha$-Aminobuttersäure und ihrer Derivate feststellen zu können, ob diese Aminosäure als Baustein der Proteine vorkommt. Bemerkenswert ist in dieser Hinsicht die von Herrn Wurm im hiesigen

1) Emil Abderhalden, Hsing Lang Chang und Erich Wurm, Synthese von Polypeptiden. Derivate der $\alpha$-Aminobuttersäure und ihr Verhalten gegenüber peptolytischen Fermenten. Diese Zeitschrift, Bd. 72, S. 24, 1911. 
Institut gemachte Beobachtung, daß aus der Aminobuttersäure auffallend leicht Ammoniak abgespalten wird. Es gilt dies ganz besonders für die Bedingungen, unter denen Eiweißkörper hydrolysiert werden. Wir werden in einer späteren Mitteilung auf diese Beobachtung zurückkommen.

Endlich sind im hiesigen Institute umfassende Untersuchungen über eine Reihe von struktur-isomeren Polypeptiden im Gange. Es sollen zunächst struktur-isomere Polypeptide dargestellt werden, die aus drei verschiedenen Aminosäuren bestehen. In unserem Falle wurden als Bausteine Glykokoll, d-Alanin und d-Aminobuttersäure gewählt. Eine eingehende Untersuchung der Eigenschaften der einzelnen Tripeptide soll einen Einblick in die Verschiedenheiten solcher relativ einfach gebauter Moleküle eröffnen. Wenn schon aus den gleichen Bausteinen bestehende Diund Tripeptide so sehr verschiedene Eigenschaften aufweisen, dann läßt sich verstehen, daß es Tausende von verschiedenartigen Proteinen gibt, die in letzter Linie die gleichen Bausteine aufweisen und zum Teil auch in gleichen Mengenverhältnissen, und sich nur durch die Reihenfolge, in der die einzelnen Bausteine sich folgen, unterscheiden. Jeder auch noch so geringfügige Unterschied in der Struktur genügt, um ganz neue Eigenschaften zu prägen. Wir werden bald über Versuche berichten können, die sich auf das biologische Verhalten von strukturisomeren Polypeptiden beziehen.

Wir haben folgende Verbindungen dargestellt:

Glycyl-dl-aminobuttersäureanhydrid; d-Aminobutyryl-glycyl-d-aminobuttersäure; d-Aminobutyrylglycyl-d-alanin; d-Aminobutyryl-d-alanin; Glycyl-daminobutyryl-d-alanin; d-Aminobutyryl-glycin; dAlanyl-d-aminobutyryl-glycin. Ferner haben wir die Waldensche Umlagerung durchgeführt.

Die folgende Tabelle gibt einen Überblick über das optische Verhalten der drei struktur-isomeren Tripeptide und der entsprechenden Halogenacylverbindungen, aus denen sie durch Aminierung entstanden sind. Die Unterschiede im Drehungsvermögen sind sehr große. Wir kommen auf diese Befunde noch eingehend zurück, wenn weiteres Material vorliegt. 


$\begin{array}{lc} & {[\alpha]_{20^{\circ}}^{\mathrm{D}}} \\ & -21,32^{\circ} \\ \text { d-Brombutyryl-glycyl-d-alanin } & -61,94^{\circ} \\ \text { Chloracetyl-d-aminobutyryl-d-alanin } & -12,83^{\circ} \\ \text { d-Brompropionyl-d-aminobutyryl-glycin } & \\ \text { d-Aminobutyryl-glycyl-d-alanin } & -7,80^{\circ} \text { (in Wasser } \\ \text { Glycyl-d-aminobutyryl-d-alanin } & -72,62^{\circ} \text { (in Welöst). Wasser } \\ & \\ \text { d-Alanyl-d-aminobutyryl-glycin } & +13,86^{\circ} \text { (in nelöst). } \\ & \text { säure gelöst). }\end{array}$

Experimenteller Teil.

Glycyl-dl-aminobuttersäureanhydrid.<smiles>CCC1NC(=O)NCC1=O</smiles>

$3,4 \mathrm{~g}$ Glycyl-dl-aminobuttersäure wurden mit $30 \mathrm{ccm}$ Methylalkohol übergossen und durch Einleiten von trockener, gasförmiger Salzsäure verestert. Nach erfolgter Sättigung der Lösung mit Salzsäure wurde noch 10 Minuten auf dem Wasserbad erwärmt und dann unter vermindertem Druck zur Trockene verdampft. Den Rückstand nahmen wir in $20 \mathrm{ccm}$ Äthylalkohol auf und leiteten in die Lösung bis zur Sättigung trockenes gasförmiges Ammoniak. Bald erfolgte Abscheidung von Chlorammon. Von diesem wurde abfiltriert und das Filtrat unter vermindertem Druck zur Trockene verdampft. Den Rückstand kochten wir wiederholt mit Essigäther aus. Beim Abkühlen der eingeengten Lösung fiel das Anhydrid krystallinisch aus. Es wurde aus heißem Wasser umkrystallisiert. Es krystallisiert aus Wasser in rhombischen Tafeln. Glycyl-dl-aminobuttersäureanhydrid ist löslich in Essigäther, Äthyl- und Methylalkohol, unlöslich in Aceton, Petroläther, absolutem Äther, Benzol, Chloroform, schwer löslich in kaltem, leichter löslich in heißem Wasser. Es schmilzt bei $238^{\circ}$. Den gleichen Schmelzpunkt geben E. Fischer 
und Raske ${ }^{1}$ ) für das aus $\alpha$-Aminobutyryl-glycin dargestellte Anhydrid an.

Zur Analyse wurde über Phosphorpentoxyd bei $100^{\circ}$ getrocknet.

$0,1080 \mathrm{~g}$ Substanz brauchten $15,30 \mathrm{ccm} 1 / 10 \mathrm{n}-$ Schwefelsäure.

$0,1560 \mathrm{~g}$ Substanz gaben $0,2905 \mathrm{~g} \mathrm{CO}_{2}$ und $0,1008 \mathrm{~g} \mathrm{H}_{2} \mathrm{O}$. Berechnet für $\mathrm{C}_{6} \mathrm{H}_{10} \mathrm{~N}_{2} \mathrm{O}_{2}(142): \mathrm{C}=50,70 \% ; \mathrm{H}=7,04 \%$; $\mathrm{N}=19,72 \%$.

Gefunden: $\mathrm{C}=50,79 \% ; \mathrm{H}=7,18 \% ; \mathrm{N}=19,85 \%$.

Umwandlung von 1-Aminobuttersäure in d-Aminobuttersäure mittels Nitrosylbromid.

$5 \mathrm{~g}$ l-Aminobuttersäure $\left([\alpha\rfloor_{20^{0}}^{\mathrm{D}}=-7,43^{\circ}\right)$ wurden mit einem Gemisch von $6 \mathrm{~g}$ Schwefelsäure und $15 \mathrm{ccm}$ Wasser übergossen. Dazu fügten wir $8 \mathrm{~g}$ Bromkali und $12 \mathrm{~g}$ Brom. In das mit Eis gekühlte Gemisch leiteten wir nun in der üblichen Weise drei Stunden lang einen kräftigen Strom von Stickoxyd ein. Dann wurden $4 \mathrm{~g}$ Brom zugegeben und das Einleiten des Stickoxds noch zwei Stunden fortgesetzt. Jetzt wurde zur Entfernung der Hauptmenge des Broms Luft durch die Lösung gesaugt und schließlich tropfenweise Natriumbisulfit zugefügt. Die entstandene Brombuttersäure wurde durch Ausäthern von der wässerigen Lösung abgetrennt, der Äther mit Chlorcalcium getrocknet und dann nach erfolgter Filtration verdunstet. Der Rückstand wurde bei $0,5 \mathrm{~mm}$ Druck destilliert. Die Brombuttersäure ging zwischen 83 und 85 über. Die Ausbeute betrug $8 \mathrm{~g}$ $=75 \%$ der Theorie.

Die so gewonnene $\alpha$-Brombuttersäure drehte bei $20^{\circ}$ in einem $0,25 \mathrm{dm}$ langen Polarisationsrohr bei Natriumlicht $5,55^{0}$ nach rechts, $d=1,439$.

$$
[\alpha]_{20^{\circ}}^{\mathrm{D}}=+15,43^{\circ} \text {. }
$$

1) Emil Fischer und Karl Raske, Derivate der $\alpha$-Aminobuttersäure. Untersuchungen über Aminosäuren, Polypeptide und Proteine, 1906, S. 510. 
Zur Überführung der $d-\alpha$-Brombuttersäure in $d-\alpha$-Aminobuttersäure wurden 3,8 $\mathrm{g}$ der Bromsäure ganz allmählich unter Eiskühlung mit $25 \%$ igem wässerigem Ammoniak übergossen. Die Lösung verblieb 4 Tage im Brutschrank. Sie wurde dann unter vermindertem Druck zur Trockene verdampft, der Rückstand in Wasser gelöst und die Lösung mit Blèioxyd gekocht. Auf diese Weise wurde das entstandene Bromammon entfernt. Aus der filtrierten Lösung fällten wir das gelöste Blei mit Schwefelwasserstoff. Dann wurde das Filtrat vom Bleisulfid durch Durchleiten von Luft vom Schwefelwasserstoff befreit und schließlich auf dem Wasserbade bis zur beginnenden Krystallisation eingeengt. Die Ausbeute an reiner d-Aminobuttersäure betrug 1,6 g $=70 \%$ der Theorie. Sie schmolz gegen $302^{\circ}$ (korr.).

Zur Analyse wurde bei $100^{\circ}$ über Phosphorpentoxyd getrocknet.

$0,1629 \mathrm{~g}$ Substanz gaben $0,2793 \mathrm{~g} \mathrm{CO}_{2}$ und $0,1260 \mathrm{~g} \mathrm{H}_{2} \mathrm{O}$. $0,1657 \mathrm{~g}$ Substanz verbrauchten $16,30 \mathrm{ccm}$ n/10-Schwefelsäure.

Berechnet für $\mathrm{C}_{4} \mathrm{H}_{9} \mathrm{NO}_{2}(103): \mathrm{C}=46,60 \% ; \mathrm{H}=8,74 \%$; $\mathrm{N}=13,59 \%$.

Gefunden: $\mathrm{C}=46,76 \% ; \mathrm{H}=8,60 \% ; \mathrm{N}=13,78 \%$.

$0,2263 \mathrm{~g}$ Substanz in Wasser gelöst. Gesamtgewicht der Lösung $6,8502 \mathrm{~g}$. $\mathrm{d}=1,006$.

$\alpha$ im $1 \mathrm{dm}$-Rohr bei Natriumlicht $0,17^{\circ}$ nach rechts.

$$
[\alpha]_{D}^{200}=+5,11^{\circ} \text {. }
$$

Es war somit Racemisierung eingetreten. Die Feststellung der Bildung von d-Aminobuttersäure aus l-Aminobuttersäure bei der Behandlung mit Nitrosylbromid bestätigt unsere frühere Beobachtung.

\section{Darstellung von d-Brombutyrylchlorid.}

$20 \mathrm{~g} \mathrm{~d}$-Brombuttersäure wurden mit $20 \mathrm{~g}$ Thionylchlorid übergossen und das Gemisch 4 Stunden bei $60^{\circ}$ des Wasserbades am Rückflußkühler erhitzt. Nach Verdampfen des überschüssigen Thionylchlorids unter vermindertem Druck bei Zimmertemperatur 
wurde das gebildete Chlorid bei $2,5 \mathrm{~mm}$ Druck destilliert. Es ging zwischen 34 und $36^{\circ}$ über. Die Ausbeute betrug $15 \mathrm{~g}=68 \%$ der Theorie. Bei $15 \mathrm{~mm}$ Druck geht das Chlorid zwischen 65 und $69^{\circ}$ über.

Wir verwandten das Brombutyrylchlorid zum Aufbau von Polypeptiden.

Darstellung optisch aktiver Di- und Tripeptide.

d-Aminobutyryl-glycyl-d-aminobuttersäure.

a) d-Brombutyryl-glycyl-d-aminobuttersäure.<smiles>CCC(Br)C(=O)NCC(=O)NC(CC)C(=O)O</smiles>

4,5 g Glycyl-d-aminobuttersäure wurden in $30 \mathrm{ccm} n-\mathrm{Na}-$ tronlauge (1 Mol.) gelöst und die Lösung abwechselnd unter guter Kühlung und Schütteln mit $6,5 \mathrm{~g} \mathrm{(11/4} \mathrm{Mol.)} \mathrm{d-Brom-}$ butyrylchlorid und $45 \mathrm{ccm} n$-Natronlauge versetzt. Die Flüssigkeit wurde dann stark angesäuert, wobei ein farbloses Öl ausfiel, welches mit Essigäther extrahiert wurde. Der Auszug wurde mit wasserfreiem Natriumsulfat getrocknet. Die filtrierte Flüssigkeit wurde im Vakuum auf ein kleines Volumen eingeengt und mit Petroläther gefällt. Die Fällung war zuerst ölig, sie wurde jedoch bald fest. Nach zweistündigem Stehen im Eisschrank wurde die Substanz abgenutscht. Das Gewicht des so erhaltenen Bromkörpers betrug 7,05 g oder $81 \%$ der Theorie. Zur Reinigung wurde die Substanz noch dreimal in Essigäther aufgenommen und mit Petroläther abgeschieden. Das so gereinigte Produkt begann bei $112^{\circ} \mathrm{zu}$ sintern und schmolz bei $139^{\circ}$ C. (korr. $141^{\circ}$ ).

Zur Analyse wurde im Vakuum über Phosphorpentoxyd bei $56^{\circ}$ bis zum konstanten Gewicht getrocknet.

0,1621 g Substanz gaben $0,2301 \mathrm{~g} \mathrm{CO}_{2}$ und $0,0823 \mathrm{~g}_{2} \mathrm{O}$. $0,1786 \mathrm{~g}$ Substanz. Verbraucht $11,70 \mathrm{ccm}$ n/10-Schwefelsäure.

0,1476 g Substanz gaben $0,0898 \mathrm{~g}$ AgBr. 
Berechnetfür $\mathrm{C}_{10} \mathrm{H}_{17} \mathrm{~N}_{2} \mathrm{O}_{4} \mathrm{Br}(309): \mathrm{C}=38,83 \% ; \mathrm{H}=5,50 \%$; $\mathrm{N}=9,06 \% ; \mathrm{Br}=25,89 \%$.

Gefunden: $\mathrm{C}=38,71 \% ; \mathrm{H}=5,64 \% ; \mathrm{N}=9,18 \%$; $\mathrm{Br}=25,89 \%$.

Die d-Brombutyryl-glycyl-d-aminobuttersäure krystallisiert aus Essigäther + Petroläther in ganz feinen Nädelchen. Sie löst sich leicht in Äthyläther, Essigäther, Aceton, absolutem Alkohol und Methylalkohol. Sie ist unlöslich in Benzol und Petroläther.

$0,5694 \mathrm{~g}$ Substanz in Wasser gelöst. Gesamtgewicht der Lösung $13,9660 \mathrm{~g}$. Spez. Gew. 1,016.

$\alpha$ im $1 \mathrm{dm}$-Rohr bei Natriumlicht: $0,23^{\circ}$ nach rechts.

$$
[\alpha]_{\mathrm{D}}^{200}=+5,55^{\circ} \text {. }
$$

b) d-A minobutyryl-glycyl-d-aminobuttersäure.

$$
\underset{\mathrm{NH}_{2}}{\mathrm{CH}_{3} \cdot \mathrm{CH}_{2} \cdot \underset{\mathrm{CH}}{\mathrm{CH}} \cdot \mathrm{CO} \cdot \mathrm{NH} \cdot \mathrm{CH}_{2} \cdot \mathrm{CO} \cdot \mathrm{NH}-\underset{\mathrm{CH}}{\mathrm{CH}} \cdot \mathrm{COOH} .}
$$

$6 \mathrm{~g}$ d-Brombutyryl-glycyl-d-aminobuttersäure wurden mit $30 \mathrm{ccm} 25 \%$ igem Ammoniak 4 Tage im Brutraum aufbewahrt. Die Lösung wurde alsdann unter vermindertem Druck eingedampft. Zur Entfernung des Chlorammoniums wurde Silbersulfat in der gewohnten Weise angewandt. Die Lösung wurde dann zunächst unter vermindertem Druck und schließlich bei gewöhnlichem Druck auf dem Wasserbade bis zur beginnenden Krystallisation eingeengt. Auf Zusatz von absolutem Alkohol fiel das Tripeptid zunächst gallertartig aus. Nach einstündigem Stehen im Eisschrank erstarrte das Produkt. Es wurde abgenutscht. Die Ausbeute betrug 3,3 g oder 70\% der Theorie. Nach mehrmaliger Reinigung durch Lösen in wenig heißem Wasser und Fällen mit Alkohol schmolz das Tripeptid bei $235^{\circ}$ (korr. $241^{\circ}$ ) unter Zersetzung.

$0,1514 \mathrm{~g}$ Substanz gaben $0,2695 \mathrm{~g} \mathrm{CO}_{2}+0,1033 \mathrm{~g} \mathrm{H}_{2} \mathrm{O}$. $0,1102 \mathrm{~g}$ Substanz verbrauchten $13,40 \mathrm{ccm}$ n/10-Schwefelsäure.

Berechnet für $\mathrm{C}_{10} \mathrm{H}_{19} \mathrm{~N}_{3} \mathrm{O}_{4}(245): \mathrm{C}=48,98 \% ; \mathrm{H}=7,76 \%$; $\mathrm{N}=17,14 \%$. 
Gefunden: $\mathrm{C}=48,55 \% ; \mathrm{H}=7,58 \% ; \mathrm{N}=17,04 \%$.

Das Tripeptid ist unlöslich in absolutem Alkohol, Methylalkohol, Essigäther, Petroläther, absolutem Äther, Aceton, Benzol, Chloroform, leicht löslich in kaltem Wasser. Es is! geschmacklos. Beim Kochen der wässerigen Lösung mit Kupferoxyd erhält man eine blau gefärbte Lösung. Auffallenderweise erhielten wir mit Triketohydrinden keine Färbung.

$0,1434 \mathrm{~g}$ Substanz in Wasser gelöst. Gesamtgewicht der Lösung 10,1934 g. Spez. Gew.: 1,003.

$\alpha$ im $1 \mathrm{dm}-$ Rohr bei Natriumlicht: $0,18^{\circ}$ nach rechts.

$$
[\alpha]_{\mathrm{D}}^{20^{\circ}}=+12,75^{\circ} \text {. }
$$

\section{4. d-Aminobutyryl-glycyl-d-alanin.}

a) d-Brombutyryl-glycyl-d-alanin.

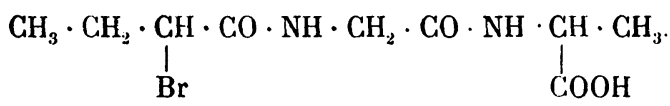

$6 \mathrm{~g}$ Glycyl-d-alanin wurden in $50 \mathrm{ccm}$ n-Natronlauge (1 Mol.) gelöst und mit $10 \mathrm{~g}$ d-Aminobutyrylchlorid (1/1/4 Mol.) und $65 \mathrm{ccm}$ Normalnatronlauge $(11 / 4 \mathrm{Mol}$.) in der üblichen Weise gekuppelt. Die ganze Operation dauerte $1 / 2$ Stunde. Nun wurde mit Salzsäure versetzt, dann die Lösung mit Essigäther extrahiert und der Auszug mit wasserfreiem Natriumsulfat getrocknet. Die filtrierte Lösung wurde im Vakuum eingeengt und mit Petroläther gefällt. Nach längerem Stehen in der Kälte fiel ein Öl aus. Nach dem Abgießen des Petroläthers wurde von neuem mit frischem Petroläther verrieben und diese Behandlung des Reaktionsproduktes noch einige Male wiederholt. Der Bromkörper schied sich schließlich flockig aus. Deutliche Krystalle waren nicht zu erkennen. Die Substanz wurde abgesaugt und mit Petroläther gewaschen. Die Ausbeute betrug $6 \mathrm{~g}=50 \%$ der Theorie.

Das d-Brombutyryl-glycyl-d-alanin löst sich leicht in Essigäther, Wasser, Aceton, absolutem Alkohol. Es ist sehr schwer löslich in Äther, unlöslich in Petroläther, in kaltem Chloroform, aber löslich in heißem. Zur Reinigung wurde die 
Substanz noch zweimal in Essigäther gelöst und mit Petroläther abgeschieden. Beim Erhitzen sintert das d-Brombutyrylglycyl-d-alanin bei $80^{\circ}$ und ist bei $145^{\circ} \mathrm{C}$. (korr. $148^{\circ}$ ) klar geschmolzen.

Zur Analyse wurde es über Phosphorpentoxyd bei $56^{\circ}$ im Vakuum bis zum konstanten Gewicht getrocknet.

$0,1794 \mathrm{~g}$ Substanz gaben $0,2396 \mathrm{~g} \mathrm{CO}_{2}$ und $0,0796 \mathrm{~g} \mathrm{H}_{2} \mathrm{O}$.

0,1805 g Substanz. Verbraucht $12,00 \mathrm{ccm} \mathrm{n/10-Schwefel-}$ säure.

$0,1263 \mathrm{~g}$ Substanz gaben $0,0810 \mathrm{~g} \mathrm{AgBr}$.

Berechnet für $\mathrm{C}_{9} \mathrm{H}_{15} \mathrm{~N}_{2} \mathrm{O}_{4} \mathrm{Br}(295): \mathrm{C}=36,61 \% ; \mathrm{H}=5,08 \%$; $\mathrm{N}=9,49 \% ; \mathrm{Br}=27,12 \%$.

Gefunden: $\mathrm{C}=36,43 \% ; \mathrm{H}=4,93 \% ; \mathrm{N}=9,32 \%$; $\mathrm{Br}=27,29 \%$.

$0,1328 \mathrm{~g}$ d-Brombutyryl-glycyl-d-alanin wurden in Wasser gelöst. Gesamtgewicht der Lösung 7,1295 g. Spez. Gew.: 1,007.

Drehung in $1 \mathrm{dm}$-Rohr $0,40^{\circ}$ nach links.

$$
[\alpha]_{\mathrm{D}}^{20^{\circ}}=-21,32^{\circ} \text {. }
$$

$$
\begin{gathered}
\text { d-Aminobutyryl-glycyl-d-alanin. } \\
\mathrm{CH}_{\mathrm{a}} \cdot \mathrm{CH}_{2} \cdot \mathrm{CH} \cdot \mathrm{CO} \cdot \mathrm{NH} \cdot \mathrm{CH}_{2} \cdot \mathrm{CO} \cdot \mathrm{NH} \cdot \mathrm{CH} \cdot \mathrm{CH}_{3} \cdot \\
\mathrm{Br}
\end{gathered}
$$

$3 \mathrm{~g}$ d-Brombutyryl-glycyl-d-alanin wurden mit $20 \mathrm{ccm}$ $25 \%$ igem Ammoniak 4 Tage im Brutraum aufbewahrt. Dann wurde die Lösung unter vermindertem Druck zur Trockene eingedampft. Das gebildete Bromammonium wurde mit Silbersulfat in der üblichen Weise entfernt und dann die Lösung auf ein kleines Volumen eingeengt. Nach Zusatz von absolutem Alkohol schied sich das Tripeptid nach kurzem Stehen gallertartig aus. Nach längerem Stehen im Eisschrank erstarrte das Produkt. Die Substanz wurde dann scharf abgesaugt und im Vakuum über Schwefelsäure getrocknet. Die Ausbeute betrug $1,4 \mathrm{~g}$ oder $61 \%$ der Theorie. Nach mehrmaligem Umfällen der Substanz aus Wasser plus absolutem Alkokol schmolz sie bei $239^{\circ}$ (korr.). Das Tripeptid ist unlöslich in allen organischen Lösungsmitteln. Seine wässerige Lösung reagiert 
auf Lackmus sauer. Sie gibt mit Triketohydrinden keine Blaufärbung.

Zur Analyse wurde es im Vakuum über Phosphorpentoxyd bei $56^{\circ}$ bis zum konstanten Gewicht getrocknet.

0,1590 g Substanz gaben $0,2731 \mathrm{~g} \mathrm{CO}_{2}$ und $0,1055 \mathrm{~g} \mathrm{H}_{\mathrm{z}} \mathrm{O}$.

$0,0996 \mathrm{~g}$ Substanz. Verbraucht $12,82 \mathrm{ccm}$ n/10-Schwefelsäure.

Berechnet für $\mathrm{C}_{9} \mathrm{H}_{17} \mathrm{~N}_{3} \mathrm{O}_{4}(231): \mathrm{C}=46,75 \% ; \mathrm{H}=7,36 \%$; $\mathrm{N}=18,18 \%$.

Gefunden: $\mathrm{C}=46,84 \% ; \mathrm{H}=7,37 \% ; \mathrm{N}=18,02 \%$.

$0,1065 \mathrm{~g}$ Substanz in Wasser gelöst. Gesamtgewicht der Lösung 6,9525 g. Spez. Gew.: 1,004.

$\alpha$ im $1 \mathrm{dm}$-Rohr bei Natriumlicht 0,12 nach links.

$$
[\alpha]_{D}^{20^{\circ}}=-7,80^{\circ} \text {. }
$$

d-Aminobutyryl-d-alanin.

a) d-Brombutyryl-d-alanin.

$$
\mathrm{CH}_{3} \cdot \mathrm{CH}_{2} \cdot \underset{\mathrm{Br}}{\mathrm{CH}} \cdot \mathrm{CO} \cdot \mathrm{NH} \cdot \underset{\mathrm{COOH}}{\mathrm{CH}} \cdot \mathrm{CH}_{3} \cdot
$$

$2 \mathrm{~g} \mathrm{~d}$-Alanin wurden in $25 \mathrm{ccm} \mathrm{n}$-Natronlauge $(1 \mathrm{Mol}$.) gelöst und in der üblichen Weise mit 4,5 g (1 1/4 Mol.) d-Brombutyrylchlorid unter Anwendung von $30 \mathrm{ccm}$ n-Natronlauge gekuppelt. Dann wurde angesäuert und mit Essigäther extrahiert. Das Extrakt wurde mit wasserfreiem Natriumsulfat getrocknet, die filtrierte Lösung unter vermindertem Druck eingeengt und dann mit Petroläther gefällt. Nach zweistündigem Stehen im Eisschrank wurden die abgeschiedenen Krystalle abgesaugt. Das Gewicht des trockenen Bromkörpers betrug $4,12 \mathrm{~g}$ oder $78 \%$ der Theorie.

d-Brombutyryl-d-alanin ist löslich in Äther, Essigäther, Aceton, absolutem Alkohol, Methylalkohol, unlöslich in Benzol, Petroläther. Es krystallisiert aus Essigäther und Petroläther teilweise in Würfelform und teilweise in dendritisch verzweigten Blättchen. Es wird gegen $110^{\circ}$ (korr. $112^{\circ}$ ) weich und schmilzt gegen $130^{\circ}$ (korr. $132^{\circ}$ ). Zur Analyse wurde es im Vakuum 
über Phosphorpentoxyd bei $56^{\circ}$ bis zum konştanten Gewicht getrocknet.

0,1708 g Substanz gaben $0,2195 \mathrm{~g} \mathrm{CO}_{2}$ und $0,0770 \mathrm{~g} \mathrm{H}_{2} \mathrm{O}$.

$0,1823 \mathrm{~g}$ Substanz. Verbraucht 7,70 ccm n/10-Schwefelsäure.

$0,2038 \mathrm{~g}$ Substanz gaben 0,1609 g AgBr.

Berechnet für $\mathrm{C}_{7} \mathrm{H}_{12} \mathrm{NO}_{3} \mathrm{Br}(238): \mathrm{C}=35,29 \% \% ; \mathrm{H}=5,05 \%$; $\mathrm{N}=5,88 \% ; \mathrm{Br}=33,61 \%$.

Gefunden: $\mathrm{C}=35,05 \% ; \mathrm{H}=5,01 \% ; \mathrm{N}=5,92 \%$; $\mathrm{Br}=33,60 \%$.

$0,5586 \mathrm{~g}$ Substanz in Wasser gelöst. Gesamtgewicht der Lösung 14,8006 g. Spez. Gew. 1,016.

$\alpha$ im $1 \mathrm{dm}$-Rohr bei Natriumlicht $0,77^{\circ}$ nach links.

$$
[\alpha]_{\mathrm{D}}^{20^{\circ}}=-20,08^{\circ}
$$

b) d-Aminobutyryl-d-alanin. $\mathrm{CH}_{3} \cdot \mathrm{CH}_{2} \cdot \underset{\mathrm{CH}}{\mathrm{i}} \cdot \mathrm{CO} \cdot \mathrm{NH} \cdot \mathrm{CH} \cdot \mathrm{CH}_{3} \cdot$

$1,6 \mathrm{~g} \mathrm{~d}$-Brombutyryl-d-alanin wurden mit $10 \mathrm{ccm} 25 \%$ igem Ammoniak 4 Tage im Brutraum aufbewahrt. Die weitere Verarbeitung war die übliche: Eindampfen unter vermindertem Druck bis zur Trockene, Lösen des Rückstandes in Wasser, Entfernen des Bromammons mit Silbersulfat und Eindampfen der Lösung bis zur Krystallisation. Sie wurde durch Zusatz von Alkohol vervollständigt. Nach zweistündigem Stehen im Eisschrank wurde die Substanz abgenutscht und im Vakuum über Schwefelsäure getrocknet. Die Ausbeute betrug 0,78 g oder 77\% der Theorie. d-Aminobutyryl-d-alanin krystallisiert aus Wasser + Alkohol in zu Büscheln vereinigten Nadeln. Das Dipeptid ist leicht löslich in Wasser, unlöslich in absolutem Alkohol, Äther, Aceton, Benzol, Essigäther, Petroläther, Chloroform. Es schmilzt bei $260^{\circ}$ (korr. 266 ${ }^{\circ}$ ). Zur Analyse wurde es im Vakuum über Phosphorpentoxyd bei $100^{\circ}$ bis zum konstanten Gewicht getrocknet.

$0,1567 \mathrm{~g}$ Substanz gaben $0,2769 \mathrm{~g} \mathrm{CO}_{2}$ und $0,1128 \mathrm{~g} \mathrm{H}_{2} \mathrm{O}$. $0,1284 \mathrm{~g}$ Substanz. Verbraucht $14,90 \mathrm{ccm} \mathrm{n} / \mathbf{1 0}_{10}$ Schwefelsäure. 


$$
\begin{aligned}
& \text { Berechnet für } \mathrm{C}_{7} \mathrm{H}_{14} \mathrm{~N}_{2} \mathrm{O}_{3}: \mathrm{C}=48,28 \% ; \mathrm{H}=8,05 \% ; \\
& \mathrm{N}=16,09 \% \text {. } \\
& \text { Gefunden: } \mathrm{C}=48,19 \% ; \mathrm{H}=8,00 \% ; \mathrm{N}=16,24 \% \text {. } \\
& \text { 0,3266 g Substanz in Normalsalzsäure gelöst. Gesamt- } \\
& \text { gewicht der Lösung } 10,3030 \text { g. Spez. Gew. 1,031. }
\end{aligned}
$$

$\alpha$ im $1 \mathrm{dm}-$ Rohr bei Natriumlicht $0,41^{\circ}$ nach links.

$$
[\alpha]_{\mathrm{D}}^{20^{\circ}}=-12,55^{\circ} \text {. }
$$

\section{Glycyl-d-aminobutyryl-d-alanin.}

a) Chloracetyl-d-aminobutyryl-d-alanin.

$$
\begin{array}{cc}
\mathrm{CH}_{3} \cdot \mathrm{CH}_{2} \cdot \mathrm{CH} \cdot \mathrm{CO} \cdot \mathrm{NH} \cdot \mathrm{CH} \cdot \mathrm{CH}_{3} \\
\mathrm{Cl} \cdot \mathrm{CH}_{2} \cdot \mathrm{CO} \cdot \mathrm{NH} & \mathrm{COOH}
\end{array}
$$

$3 \mathrm{~g}$ d-Aminobutyryl-d-alanin wurden in $25 \mathrm{ccm} \mathrm{n}$-Natronlauge (1 Mol.) gelöst und die Lösung unter starker Kühlung und Schütteln mit $3 \mathrm{~g}$ (1,5 Mol.) Chloracetylchlorid und $40 \mathrm{ccm}$ n-Natronlauge abwechselnd versetzt. Dann wurde angesäuert. Da keine Abscheidung von Krystallen erfolgte, wurde mit Essigäther extrahiert und das Extrakt mit wasserfreiem Natriumsulfat getrocknet. Nun wurde die filtrierte Lösung unter vermindertem Druck auf ein kleines Volumen eingeengt und mit Petroläther gefällt. Die, Fällung wurde nach mehrstündigem Stehen im Eisschrank abgesaugt. Die Gesamtausbeute betrug $3,3 \mathrm{~g}$ oder $77 \%$ der Theorie.

Chloracetyl-d-aminobutyryl-d-alanin krystallisiert aus Essigäther + Petroläther in Nadeln. Es schmilzt bei $190^{\circ}$ (korr. $195^{\circ}$ ). Die Verbindung ist in Wasser, Essigäther, Äther, absolutem Alkohol, Aceton löslich, unlöslich in Benzol, Petroläther und Chloroform. Zur Analyse wurde der Körper im Vakuum über Phosphorpentoxyd bei $100^{\circ}$ bis zum konstanten Gewicht getrockent.

0,1805 g Substanz gaben $0,2875 \mathrm{~g} \mathrm{CO}_{2}$ und $0,0969 \mathrm{~g} \mathrm{H}_{2} \mathrm{O}$.

$0,1290 \mathrm{~g}$ Substanz. Verbraucht $10,55 \mathrm{~cm}$ n/10-Schwefelsäure.

$0,1486 \mathrm{~g}$ Substanz gaben $0,0858 \mathrm{~g} \mathrm{AgCl}$.

Berechnet für $\mathrm{C}_{9} \mathrm{H}_{15} \mathrm{~N}_{2} \mathrm{O}_{4} \mathrm{Cl}(250,5): \mathrm{C}=43,11 \% ; \mathrm{H}$ $=5,99 \% ; \mathrm{N}=11,18 \%$ Cl $=14,17 \%$. 
Gefunden: $\mathrm{C}=43,44 \% ; \mathrm{H}=5,97 \% ; \mathrm{N}=11,46 \%$; $\mathrm{Cl}=14,28 \%$.

$0,1264 \mathrm{~g}$ Substanz in Wasser gelöst. Gesamtgewicht der Lösung 6,1902 g. Spez. Gew. 1,028.

$\alpha$ im $1 \mathrm{dm}$-Rohr bei Natriumlicht: $-1,30^{\circ}$ nach links.

$$
[\alpha]_{D}^{200}=-61,94^{\circ} .
$$

b) Glycyl-d-aminobutyryl-d-alanin.

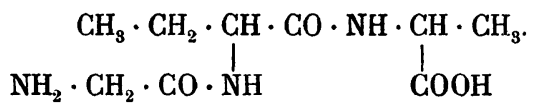

$1,5 \mathrm{~g}$ Chloracetyl-d-aminobutyryl-d-alanin wurden mit $15 \mathrm{ccm} 25 \%$ igem Ammoniak 4 Tage im Brutschrank aufbewahrt. Auch hier wurde nach Entfernung des gebildeten Chlorammons mit Silbersulfat bis zur beginnenden Substanzabscheidung eingeengt. Sie wurde durch Zusatz von Alkohol vervollständigt. Das Tripeptid fällt zunächst gallertartig aus. Erst nach längerem Stehen im Eisschrank ließ es sich abnutschen. Es wurde im Vakuum über Schwefelsäure getrocknet. Die Gesamtausbeute betrug $1 \mathrm{~g}$ oder $70 \%$ der Theorie. Es reagiert in wässeriger Lösung neutral auf Lackmus. Die Löslichkeit ist ähnlich, wie beim d-Aminobutyryl-glycyl-d-alanin. Es schmilzt bei $240-241^{\circ}$ C. (korr. $247^{\circ}$ ). Zur Analyse wurde es im Vakuum über Phosphorpentoxyd bei $56^{\circ}$ bis zum konstanten Gewicht getrocknet.

$0,1582 \mathrm{~g}$ Substanz gaben $0,2694 \mathrm{~g} \mathrm{CO}_{2}$ und $0,1062 \mathrm{~g} \mathrm{H}_{2} \mathrm{O}$.

$0,0963 \mathrm{~g}$ Substanz. Verbraucht $12,65 \mathrm{ccm} \mathrm{n} / 10-S c h w e f e l-$ säure.

Berechnet für $\mathrm{C}_{9} \mathrm{H}_{17} \mathrm{~N}_{3} \mathrm{O}_{4}(231): \mathrm{C}=46,75 \% ; \mathrm{H}=7,36 \%$; $\mathrm{N}=18,18 \%$.

Gefunden: $\mathrm{C}=46,44 \% ; \mathrm{H}=7,46 \% ; \mathrm{N}=18,40 \%$.

$0,1287 \mathrm{~g}$ Substanz in Wasser gelöst. Gesamtgewicht der Lösung 6,9340 g. Spez. Gew. 1,006.

$\alpha$ im $1 \mathrm{dm}-$ Rohr $1,43^{\circ}$ nach links.

$$
[\alpha]_{\mathrm{D}}^{200}=-76,62^{\circ} .
$$

Die wässerige Lösung reagiert sauer und gibt mit Triketohydrindenhydrat keine Blaufärbung. 
d-Aminobutyryl-glycin.

a) d-Brombutyryl-gly cin.
$\mathrm{CH}_{3} \cdot \mathrm{CH}_{2} \cdot \underset{\mathrm{Br}}{\mathrm{CH}} \cdot \mathrm{CO} \cdot \mathrm{NH} \cdot \mathrm{CH}_{2} \cdot \mathrm{COOH}$.

$4 \mathrm{~g}$ Glykokoll wurden in $55 \mathrm{ccm} \mathrm{n}$-Natronlauge (1 Mol.) gelöst und $\mathrm{zu}$ der mit einer Kältemischung gekühlten Flüssigkeit in der üblichen Weise $11,5 \mathrm{~g}$ d-Brombutyrylchlorid (11/4 Mol.) und $75 \mathrm{ccm} n$-Natronlauge abwechselnd zugesetzt. Die ganze Operation dauerte ${ }^{1 / 2}$ Stunde. Nach erfolgtem Ansäuern wurde die Lösung mit Äther oder Essigäther extrahiert und das Extrakt mit wasserfreiem Natriumsulfat getrocknet. Die filtrierte Lösung wurde dann eingedampft und der Rückstand mit Petroläther gefällt. Das Gewicht des abgesaugten Bromkörpers betrug $8 \mathrm{~g}$ oder $70^{\%}$ der Theorie. Er schmilzt gegen $92^{\circ} \mathrm{C}$. (korr. $93^{\circ}$ ) und ist löslich in absolutem Äther, Essigäther, Wasser, Aceton, absolutem Alkohol, schwer löslich in kaltem Benzol, aber leicht in heißem Benzol, unlöslich in Petroläther. Der Bromkörper wurde mehrmals in Äther gelöst und mit Petroläther gefällt. Zur Analyse wurde er im Vakuum über Phosphorpentoxyd bei $56^{\circ}$ bis zum konstanten Gewicht getrocknet.

$0,1728 \mathrm{~g}$ Substanz gaben $0,2044 \mathrm{~g} \mathrm{CO}_{2}$ und $0,0769 \mathrm{~g} \mathrm{H}_{2} \mathrm{O}$. $0,1346 \mathrm{~g}$ Substanz. Verbraucht $6,00 \mathrm{ccm}^{\mathrm{n}} / 10-$ Schwefelsäure. $0,1238 \mathrm{~g}$ Substanz gaben $0,1034 \mathrm{~g} \mathrm{AgBr}$.

Berechnet für $\mathrm{C}_{6} \mathrm{H}_{10} \mathrm{NO}_{3} \mathrm{Br}(224): \mathrm{C}=32,14 \% \%_{0} ; \mathrm{H}=4,46 \%$; $\mathrm{N}=6,25 \% ; \mathrm{Br}=35,71 \%$.

Gefunden: $\mathrm{C}=32,26 \% ; \mathrm{H}=4,95 \% ; \mathrm{N}=6,25 \% ; \mathrm{Br}$ $=35,54 \%$.

0,4982 g Substanz in Wasser gelöst. Gesamtgewicht der Lösung 7,3093 g. Spez. Gew. 1,018.

$\alpha$ im $1 \mathrm{dm}-$ Rohr bei Natriumlicht 2,25 nach rechts.

$$
[\alpha]_{\mathrm{D}}^{20^{\circ}}=+32,44^{\circ} \text {. }
$$

b) d-Aminobutyryl-glycin.

$\mathrm{CH}_{3} \cdot \mathrm{CH}_{2} \cdot \mathrm{CH} \cdot \mathrm{CO} \cdot \mathrm{NH} \cdot \mathrm{CH}_{2} \cdot \mathrm{COOH}$.

$\stackrel{1}{\mathrm{NH}_{2}}$

$5 \mathrm{~g}$ d-Brombutyryl-glycin wurden mit $30 \mathrm{ccm} 25 \%$ igem Ammoniak im Brutschrank 4 Tage aufbewahrt. Nach der üb- 
lichen Entfernung des Bromammons mit Silbersulfat wurde auf ein kleines Volumen eingeengt und mit absolutem Alkohol versetzt. Das ausgefallene Dipeptid wurde dann nach einigem Stehen bei $0^{\circ}$ abgenutscht und im Vakuum über Schwefelsäure getrocknet. Die Ausbeute betrug 2,2 g oder $62 \%$ der Theorie. Es schmilzt gegen $221^{\circ}$ C. (korr. $226^{\circ}$ ). Es scheidet sich aus absolutem Alkohol und Wasser als krystallinisches Pulver ab. Zur Analyse wurde es im Vakuum bei $100^{\circ}$ über $\mathrm{P}_{2} \mathrm{O}_{5}$ bis zum konstanten Gewicht getrocknet.

$0,1952 \mathrm{~g}$ Substanz. Verbraucht $24,50 \mathrm{ccm} \mathrm{n/10-Schwefel-}$ säure.

Berechnet für $\mathrm{C}_{6} \mathrm{H}_{12} \mathrm{~N}_{2} \mathrm{O}_{3}(160): \mathrm{N}=17,50 \%$.

Gefunden: $N=17,58 \%$.

Alle Bemühungen, stimmende Werte für $\mathrm{C}$ zu erhalten, schlugen fehl. Die Differenz wurde beim Umkrystallisieren immer größer. Sie betrug für $\mathrm{C}$ ca. 1-2\%. Der Wasserstoffwert und der Stickstoffwert stimmte stets. Es scheint, daß entweder bei der Reinigung eine Veränderung des Dipeptids vor sich geht, oder aber die Analyse birgt Fehler in sich. Wir haben das d-Aminobutyryl-glycin wiederholt dargestellt und immer die gleichen Analysenresultate erhalten. Der Umstand, da $B$ aus diesem Dipeptid beim Kuppeln mit d-Brompropionylchlorid und nachfolgender Aminierung ein Tripeptid entstand, das richtige Analysenwerte ergab, weist darauf hin, daß das verwendete Dipeptid rein war. Mit der Aufklärung des merkwürdigen Analysenbefundes sind wir noch beschäftigt.

$0,1278 \mathrm{~g}$ Substanz in Wasser gelöst. Gesamtgewicht der Lösung $8,0325 \mathrm{~g}$. Spez. Gew. 1,007.

$\alpha$ im $1 \mathrm{dm}$-Rohr bei Natriumlicht $0,43^{\circ}$ nach rechts.

$$
[\alpha]_{\mathrm{D}}^{20^{\circ}}=+26,83^{\circ} \text {. }
$$

d-Alanyl-d-aminobutyryl-glycin.

a) d-Brompropionyl-d-aminobutyryl-glycin.

$$
\begin{array}{cc}
\mathrm{CH}_{3} \cdot \underset{1}{\mathrm{CH}}-\mathrm{CO}-\mathrm{NH}-\mathrm{CH}-\mathrm{CH}_{2}-\mathrm{CH}_{3} . \\
\mathrm{Br} & \mathrm{CO}-\mathrm{NH}-\mathrm{CH}_{2} \cdot \mathrm{COOH} .
\end{array}
$$

2,35 g d-Aminobutyryl-glycin wurden in $20 \mathrm{ccm} n$-Natronlauge (1 Mol.) gelöst und unter guter Kühlung und Schütteln 
mit 3,5 $\mathrm{g} \mathrm{d}$-Brompropionylchlorid (11/4 Mol.) und $30 \mathrm{ccm} \mathrm{n}$-Natronlauge $\left(1^{1 / 2}\right.$ Mol.) gekuppelt. Nach erfolgtem Ansäuren wurde mit Essigäther extrahiert, der Auszug mit geglühtem Natriumsulfat getrocknet und dann die filtrierte Lösung im Vakuum eingeengt und der Rückstand mit Petroläther gefällt. Der Bromkörper bildete jetzt eine weiße, rasch fest werdende Masse. Aus dem Gemisch von Essigäther + Petroläther krystallisiert der Bromkörper in langen Nadeln. Die Gesamtausbeute betrug $3,05 \mathrm{~g}$ oder $70 \%$ der Theorie. Zur Reinigung wurde die Substanz zweimal aus Essigäther + Petroläther umkrystallisiert.

Der Bromkörper ist in Äther, Essigäther, absolutem Alkohol, Methylalkohol, Aceton und Wasser löslich, unlöslich in Petroläther. Das d-Brompropionyl-d-aminobutyryl-glycin schmilzt bei $163^{\circ}$ (korr. $166^{\circ}$ ). Zur Analyse wurde es im Vakuum über Phosphorpentoxyd bei $56^{\circ}$ getrocknet.

$0,1291 \mathrm{~g}$ Substanz. Verbraucht $9,03 \mathrm{ccm}{ }^{\mathrm{n}} / \mathbf{1 0}-\mathrm{Sch}$ wefelsäure.

$0,1675 \mathrm{~g}$ Substanz gaben $0,1080 \mathrm{~g} \mathrm{AgBr}$.

$0,1721 \mathrm{~g}$ Substanz gaben $0,2314 \mathrm{~g} \mathrm{CO}_{2}$ und $0,0765 \mathrm{~g} \mathrm{H}_{2} \mathrm{O}$.

Berechnet für $\mathrm{C}_{9} \mathrm{H}_{15} \mathrm{~N}_{2} \mathrm{O}_{4} \mathrm{Br}$ (295): $\mathrm{C}=36,61 \%$ : H $=5,08 \% ; \mathrm{N}=9,49 \% ; \mathrm{Br}=27,12 \%$.

Gefunden: $\mathrm{C}=36,67 \% ; \mathrm{H}=4,94 \% ; \mathrm{N}=9,60 \%$; $\mathrm{Br}=27,44 \%$.

Zur optischen Bestimmung wurde der Bromkörper in Wasser gelöst.

0,1081 g Substanz. Gesamtgewicht der Lösung 7,3311 g. Spez. Gew. 1,004. Drehung im $1 \mathrm{dm}$-Rohr bei Natriumlicht: $0,19^{\circ}$ nach links.

$$
[\alpha]_{\mathrm{D}}^{200}=-12,83^{\circ} .
$$

b) d-Alanyl-d-aminobutyryl-glycin.

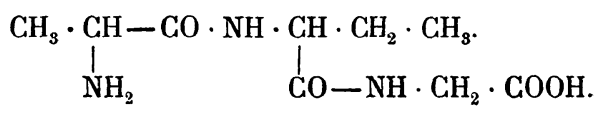

$1,8 \mathrm{~g}$ d-Brompropionyl-d-aminobutyryl-glycin wurden mit $10 \mathrm{ccm} 25 \%$ igem Ammoniak 4 Tage im Brutraum aufbewahrt. Das Bromammonium wurde aus der eingedampften Lösung nach 
Aufnahme des Rückstandes in Wasser mit Silbersulfat entfernt. Das Tripeptid fällt aus der eingeengten wässerigen Lösung auf Zusatz von Alkohol gallertartig aus. Es wurde nach einigem Stehen fest und konnte dann auf einer Nutsche abgesaugt werden. Die im Vakuum über Schwefelsäure getrocknete Substanz wog $1 \mathrm{~g}$. Zur Reinigung wurde sie noch dreimal in Wasser aufgenommen und mit absolutem Alkohol abgeschieden.

Das Tripeptid schmilzt bei $210^{\circ}$ (korr. 2140) und ist in allen organischen Lösungsmitteln unlöslich. Zur Analyse wurde über $\mathrm{P}_{8} \mathrm{O}_{5}$ bei $56^{\circ}$ getrocknet.

$0,1550 \mathrm{~g}$ Substanz gaben $0,2650 \mathrm{~g} \mathrm{CO}_{2}$ und 0,1012 $\mathrm{g} \mathrm{H}_{2} \mathrm{O}$. $0,1080 \mathrm{~g}$ Substanz. Verbraucht $14,05 \mathrm{ccm}$ n/10-Schwefelsäure.

Berechnet für $\mathrm{C}_{9} \mathrm{H}_{17} \mathrm{~N}_{3} \mathrm{O}_{4}(231): \mathrm{C}=46,75 \% ; \mathrm{H}=7,36 \%$; $\mathrm{N}=18,18 \%$.

Gefunden: $\mathrm{C}=46,63 \% ; \mathrm{H}=7,26 \% ; \mathrm{N}=18,23 \%$. $0,0562 \mathrm{~g}$ Substanz in Normalsalzsäure gelöst. Gesamtgewicht der Lösung 7,2198 g. Spez. Gew.: 1,02.

$\alpha$ im $1 \mathrm{dm}$-Rohr bei Natriumlicht: $0,11^{0}$ nach rechts.

$$
[\alpha]_{\mathrm{D}}^{20^{\circ}}=+13,86^{\circ} \text { in } \mathrm{n} / 1-\mathrm{HCl} \text {. }
$$

Die wässerige, sauer reagierende Lösung gibt mit Triketohydrindenhydrat keine Blaufärbung. Auf Zusatz einer Spur 1/10-Natronlauge erfolgt Gelbfärbung. Diese geht rasch in Braunund dann in Grünfärbung über.

\section{Anhang.}

Die in der 1. Mitteilung beschriebene Glycyl-d-aminobuttersäure war optisch nicht ganz rein, wie schon eine Vergleichung des gefundenen Drehungsvermögens mit dem der Glycyl-l-aminobuttersäure ergab. Wir haben dieses Dipeptid nochmals dargestellt und fanden $[\alpha]_{D}^{200}=-20,33^{\circ}$.

$0,4416 \mathrm{~g}$ Substanz in Wasser gelöst. Gesamtgewicht der Lösung 8,3544 g. $\mathrm{d}=1,014$. $\alpha$ im $1 \mathrm{dm}$-Rohr bei Natriumlicht $1,09^{\circ}$ nach links. 